\title{
Combination Method for Powerline Interference Reduction in ECG
}

\author{
Manpreet Kaur \\ Deptt of EIE \\ SLIET Longowal \\ Dist Sangrur $(\mathrm{Pb})$ India
}

\author{
A.S.Arora \\ Professor, Deptt of EIE \\ SLIET Longowal \\ Dist Sangrur $(\mathrm{Pb})$ India
}

\begin{abstract}
The ECG signal is a graphical representation of the electromechanical activity of the cardiac system. For diagnostic quality ECG recordings, signal acquisition must be noise free. Since ECG signals are only of the order of $1 \mathrm{mV}$ in amplitude, the ECG acquisition is susceptible to many types of noises In fact the waveforms of interest are sometimes so heavily masked by noise that their presence can only be revealed after the applications of appropriate signal processing tools. Common to all types of ECG analysis - whether it concerns resting ECG interpretation, stress testing, ambulatory monitoring or intensive care monitoring the first step is to remove the noise and Powerline interference is one of them. Many types of analog and Digital Filters have been proposed/ suggested by the researchers. The present paper has proposed a combination technique of three methods i.e. the Moving Averages technique, IIR Notch and Wavelets to reduce the Powerline interference. The results have clearly indicated that there is reduction in Powerline noise in the ECG signal. The results have been concluded using Matlab and MIT-BIH database (with $60 \mathrm{~Hz}$ Powerline interference)
\end{abstract}

\section{Categories and Subject Descriptors}

Filtering and Signal Processing of ECG Signal

\section{General Terms}

ECG, Powerline Interference, Moving Averages, IIR Notch, Wavelets, MIT-BIH, Noise, ECG Filtering, Coiflet, Harr, Symmlet, Daubechies, denoising, Power Spectral Density, Baseline wander, QRS, Drifting, FDATool

\section{Keywords}

Combination Method, PSD

\section{INTRODUCTION TO ECG SIGNAL}

The ECG signal is represented as PQRST waveform The first phase of cardiac muscle activation is the stimulation of the right and left atria by an electrical signal generated from SA node. The depolarization of atria appears as the P-wave on the ECG waveform. The electrical signal generated, originally generated by the SA node, then spreads through the Atrioventricular (AV) node, the bundle of HIS, and the Purkinje fibers to finally reach and stimulate the ventricles. The spread of electrical signal through ventricles causes ventricular contraction. The phase of repolarization of atria and depolarization of ventricles appears as the characteristic QRS complex on ECG waveform. Finally, the repolarization of two ventricles appears as the T-wave on ECG waveform.[1]

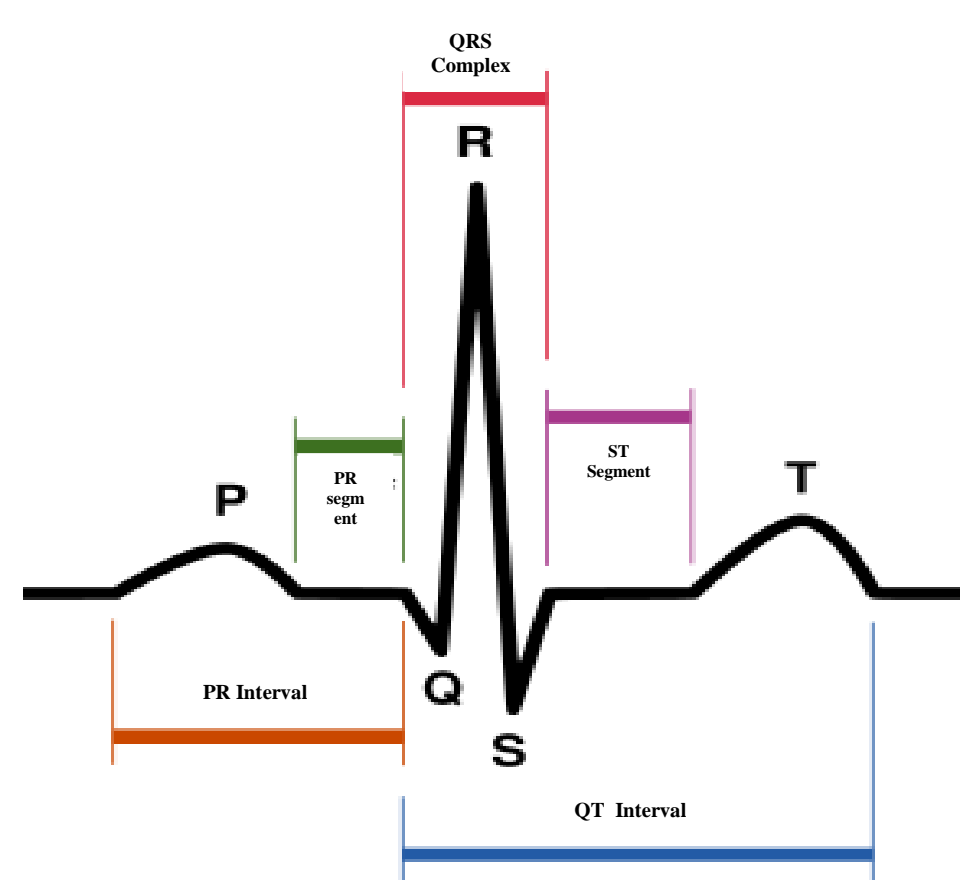

Fig 1 : The ECG Waveform[2]

\section{THE NOISES IN ECG SIGNAL}

The common noise sources are :

- Baseline wander and ECG amplitude modulation with respiration

- $\quad$ Power line interference

- Muscle contraction noise

- Electrosurgical noise

- Motion artifacts

- Noise due to variation of electrode skin contact impedance

- Noise generated by electronic devices used in signal processing circuits

Many Signal processing tools are used now-a-days for analysis and interpretation of ECG signal. The very first step in any of the ECG processing is the noise reduction and Powerline noise reduction is one of it. 
Powerline interference noise is electromagnetic field from the Powerline which causes $50 / 60 \mathrm{~Hz}$ sinusoidal interference. This noise causes problem in interpreting low amplitude waveform like ECG. Although, various measures like shielding and grounding are taken into care while recording ECG signal yet the cables are susceptible to electromagnetic interference. In order to avoid the wrong identification of the characteristics of ECG signals and its impact on analysis and diagnosis accuracy, it is necessary to remove the power-line interference effectively.

\section{ECG FILTERING}

The analysis of ECG signal has been widely used for diagnosing many cardiac diseases. ECG signal provides almost all the information about electrical activity of the heart so care should be taken while doing the ECG filtering, such that the desired information is not distorted or altered in any way. Researchers have worked on the removal of the Powerline interference in the ECG signals. Many methods have been suggested or proposed.

Alireza K Ziarani,Adaibert Konrad[3]have proposed Non linear Adaptive method of elimination of power line interference in ECG signals. S.Pooranchandra, N.kumarave [4] have used the wavelet coefficient threshold based hyper shrinkage function to remove power line frequency. Santpal Singh Dhillon and Saswat Chakrabarti [5] have used a simplified lattice based adaptive IIR Notch filter to remove power line interference. Mahesh S. Chavan, R.A. Aggarwala, M.D.Uplane [6] have used Digital FIR Filters based on Rectangular window for the powerline noise reduction. ChavdarLevkov, Georgy Mihov, Ratcho Ivanov, Ivan Daskalov, Ivaylo Christov, and Ivan Dotsinsky [7] have used the subtraction procedure to remove the powerline noise Ziarani AK, KonardA [8] used a nonlinear adaptive method to remove noise. LIN Yue-Der, YU HEN HU [9] have proposed a PLI detector that employs an optimal linear discriminant analysis (LDA). G. Mihov, Iv Dotsinsky, Ts Georgieva [10] have proposed subtraction procedure for powerline interference removing from ECG which is extended to almost all possible cases of sampling rate and interference frequency variation. K.Daqrouq [11] has removed ECG baseline wandering using Discrete Wavelet Transform. P.E.Tikkane [12] has applied Non linear wavelet and wavelet packet for denoising of electrocardiogram signal.

\section{COMBINATION METHOD: PROPOSED TECHNIQUE}

A major source of interference in ECG signals is the $50 / 60 \mathrm{~Hz}$ power line frequency. The $60 \mathrm{~Hz}$ frequency component can be removed by using IIR notch filter. The sharpness of the notch may be improved by placing a few poles near or symmetrically around the zeros and inside the unit circle. But the increased transient response time results in a ringing artifact after the transient.

The general, more sophisticated filters can be constructed by having a narrower notch but it is not possible to design an IIR notch to remove the noise without causing ringing.[13]

So a combination technique has been proposed /suggested to filter out $50 / 60 \mathrm{~Hz}$ power line interference in this paper. In this proposed method, the moving average algorithm is applied to the ECG signal in first step, afterwards the IIR notch is applied and then Wavelets are applied in the third stage and signal is denoised using Wavelets (db4).

\subsection{Moving Average Method}

Moving Averages algorithm is applied in cases where a given waveform is cluttered with noise, where a mean needs to be extracted from a periodic signal or where a slowly drifting baseline needs to be eliminated from a higher frequency signal. A moving average filter smoothes data by replacing each data point with the average of the neighboring data points defined within the span the algorithm accomplishes a moving average by taking two or more number of data points from the waveform data, adding them, dividing their sum by the total number of data points added, replacing the first data point of the waveform with the average just computed and repeating the steps with the second, third and so on data points until the end of the data is reached. The result is a second or generated waveform consisting of the averaged data and having the same number of points as the original waveform The appropriate smoothing factor has been taken determined by dividing one waveform period with the channel's sample interval [14]. The proposed work has taken twice the above ratio. This results in less variations in the resultant ECG signal.

\subsection{IIR Notch}

The filter is designed taking care that lesser ringing effect is introduced and the desired information is not altered in any way. The filter characteristics have been shown using FDATool design box, as below:

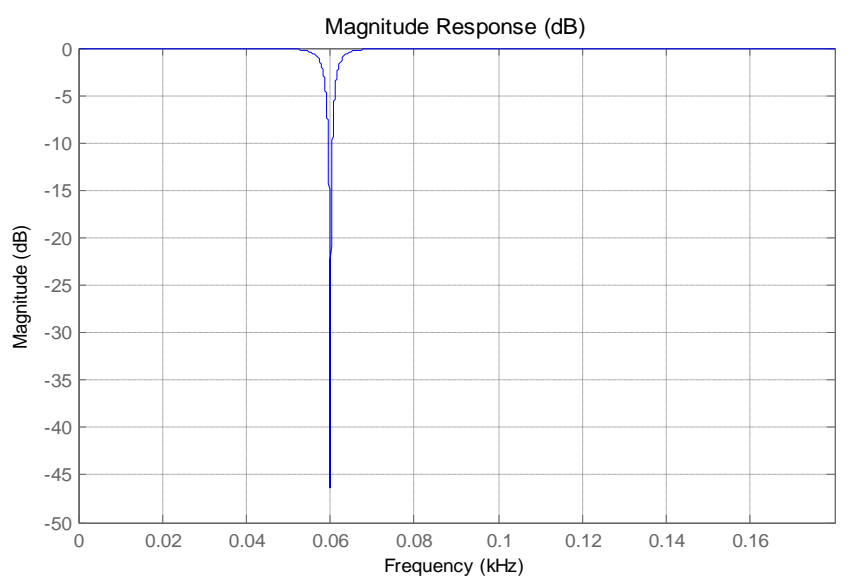

Fig 1.2: Magnitude response 


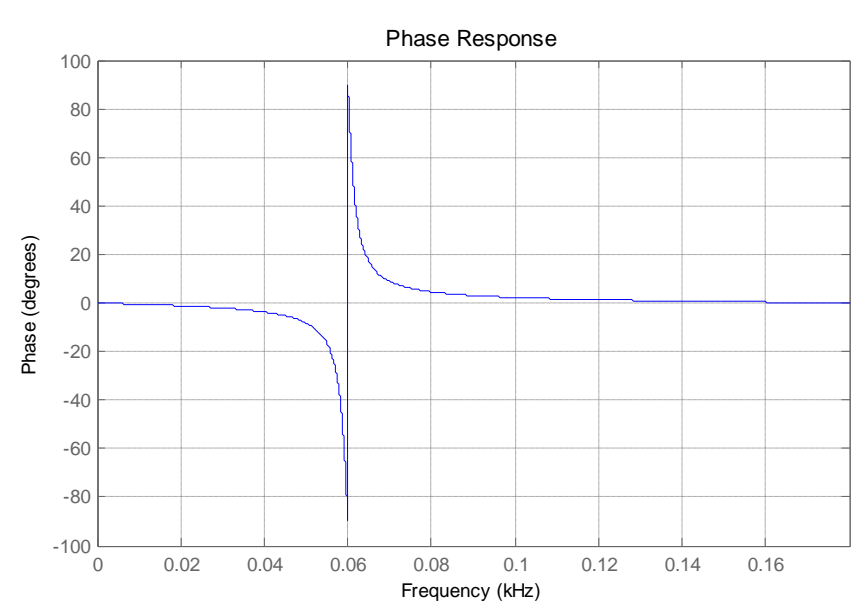

Fig 1.3: Phase response

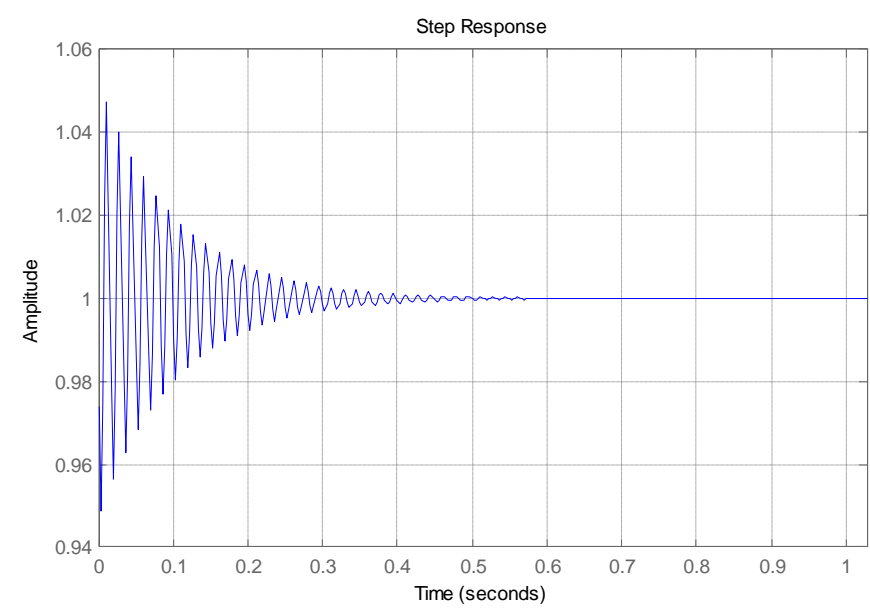

Fig 1.4: Step response

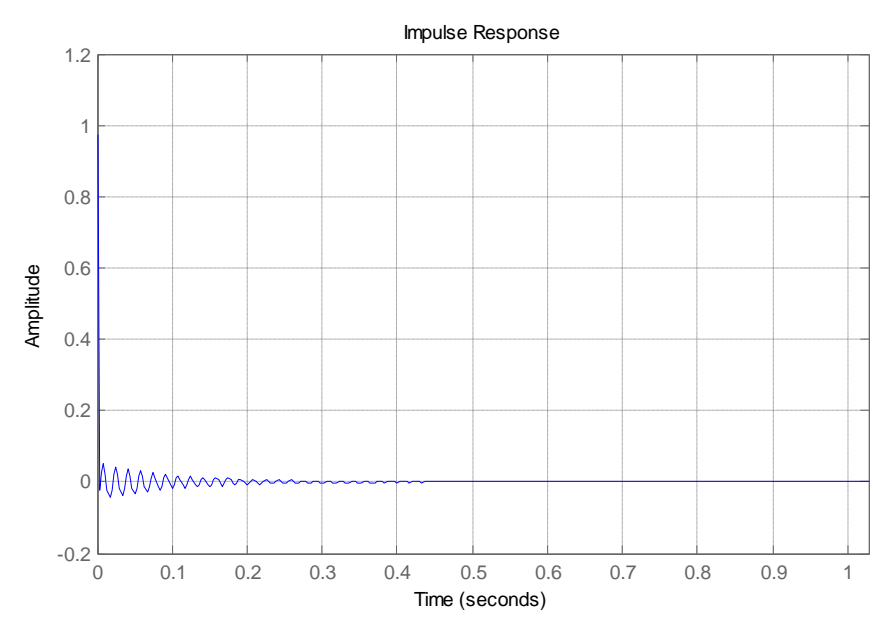

Fig 1.5: Impulse response

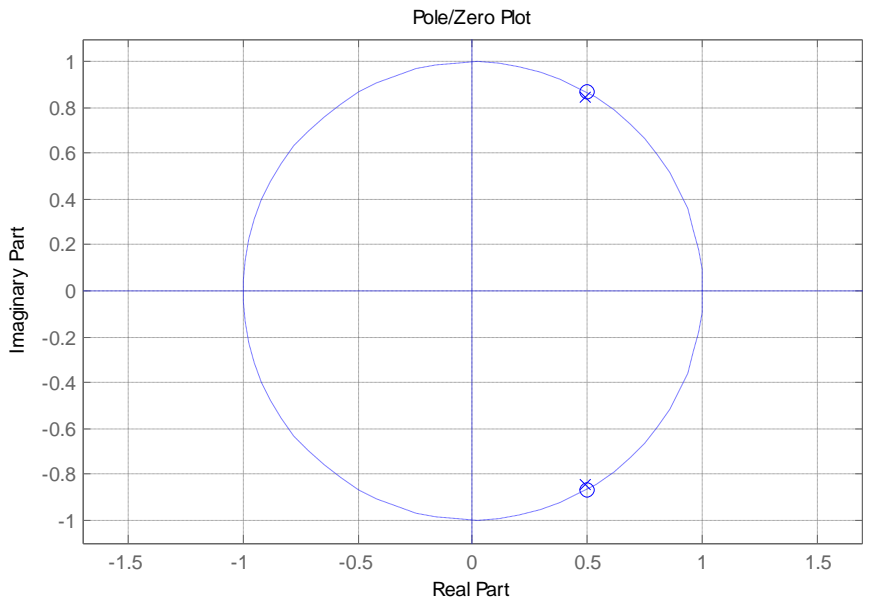

Fig 1.6: Pole Zero Plot

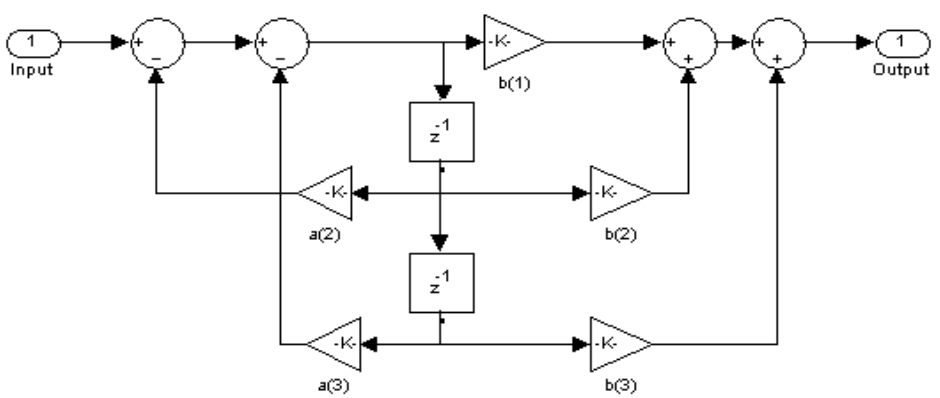

Fig 1.7: The Filter realisation

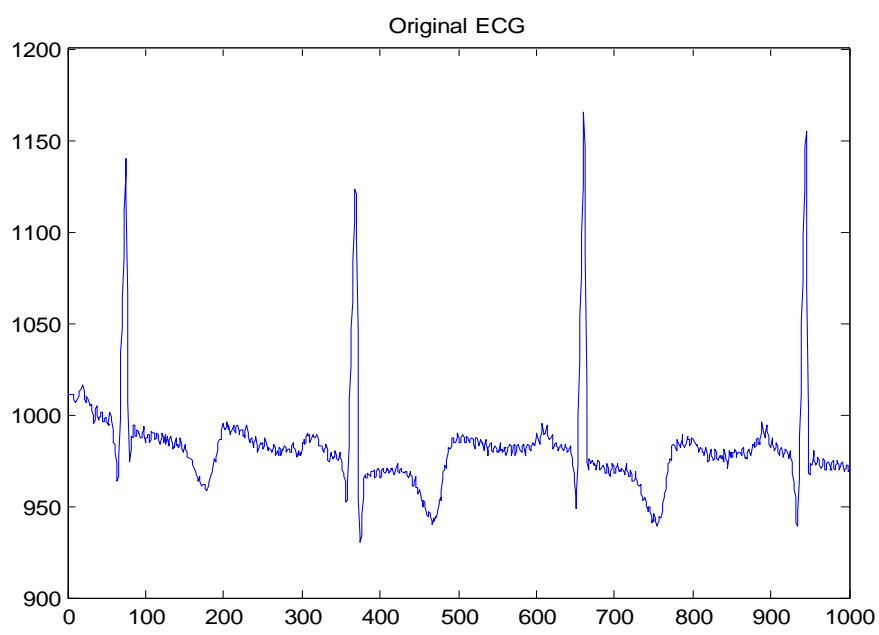

Fig 1.8: The original ECG signal 


\begin{tabular}{|l|l|l|l|}
\hline $\begin{array}{l}\text { S } \\
\text { No }\end{array}$ & $\begin{array}{l}\text { Sample } \\
\text { Data }\end{array}$ & $\begin{array}{l}\text { PSD after } \\
\text { Baseline } \\
\text { Drift }\end{array}$ & $\begin{array}{l}\text { PSD of Denoised ECG } \\
\text { (After removing Baseline } \\
\text { Drift \& without applying } \\
\text { Notch) }\end{array}$ \\
\hline 1 & Sample 1 & 23.96577 & 9.424418 \\
\hline 2 & Sample 2 & 18.19947 & 6.528052 \\
\hline 3 & Sample 3 & 22.36831 & -4.684642 \\
\hline 4 & Sample 4 & 23.62872 & 13.27538 \\
\hline 5 & Sample 5 & 27.40474 & 16.13177 \\
\hline 6 & Sample 6 & 24.41057 & 19.20307 \\
\hline
\end{tabular}

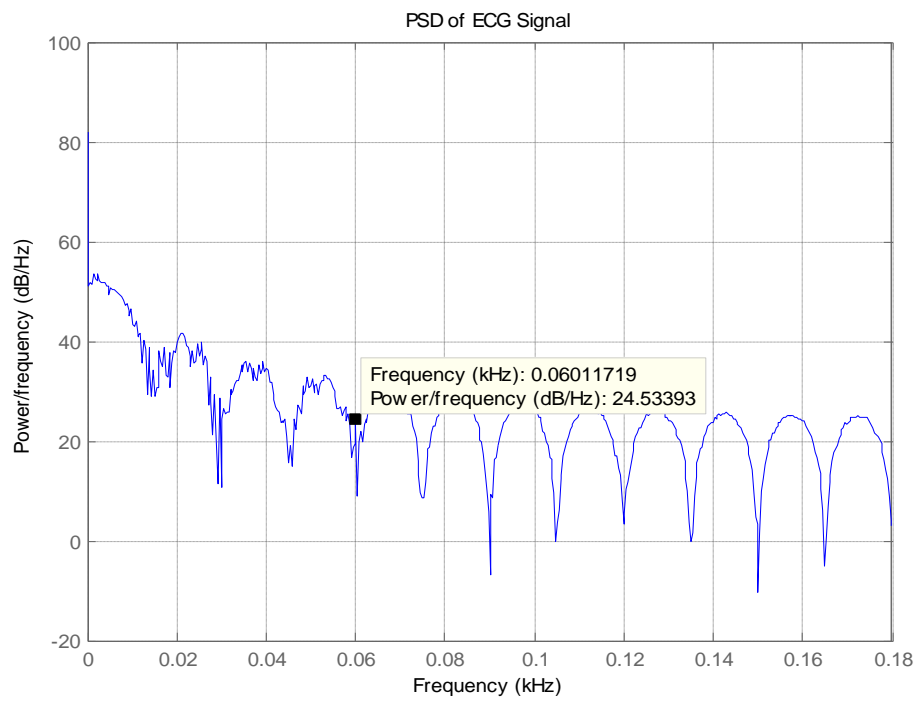

Fig 1.9: PSD of original signal

\subsection{Wavelets}

Wavelets have emerged as one of the most powerful tool for denoising of ECG signals.Large number of known wavelets families and functions like Coiflet, Harr, Symmlet, Daubechies etc are available for analysis. Selecting a wavelet function which closely matches the signal to be processed is of utmost importance in wavelets applications. Since the Daubechies wavelet family are similar in shapre to QRS comlplex and their energy spectrum are concentrated around low frequency, hence $\mathrm{db}$ is used for current analysis [15].

\section{RESULTS}

The results are obtained by taking the data of sample signals available in BIT-MIH database and shown below are the results of a sample signal. The work is carried out in three steps: in the first step, Wavelets are applied on the sample ECG signals and PSD of the sample ECG and denoised ECG are compared as shown in the Table1.
Table 1:

\begin{tabular}{|l|l|l|l|}
\hline $\begin{array}{l}\text { S } \\
\text { No }\end{array}$ & $\begin{array}{l}\text { Sample } \\
\text { Data }\end{array}$ & $\begin{array}{l}\text { PSD of } \\
\text { original Signal }\end{array}$ & $\begin{array}{l}\text { PSD after applying } \\
\text { Wavelets directly }\end{array}$ \\
\hline 1 & Sample 1 & 24.53393 & 11.61887 \\
\hline 2 & Sample 2 & 19.46361 & 3.352992 \\
\hline 3 & Sample 3 & 22.90612 & 3.270722 \\
\hline 4 & Sample 4 & 23.70606 & 12.6825 \\
\hline 5 & Sample 5 & 29.66515 & 23.19517 \\
\hline 6 & Sample 6 & 23.61126 & 16.63417 \\
\hline
\end{tabular}

Table 2:
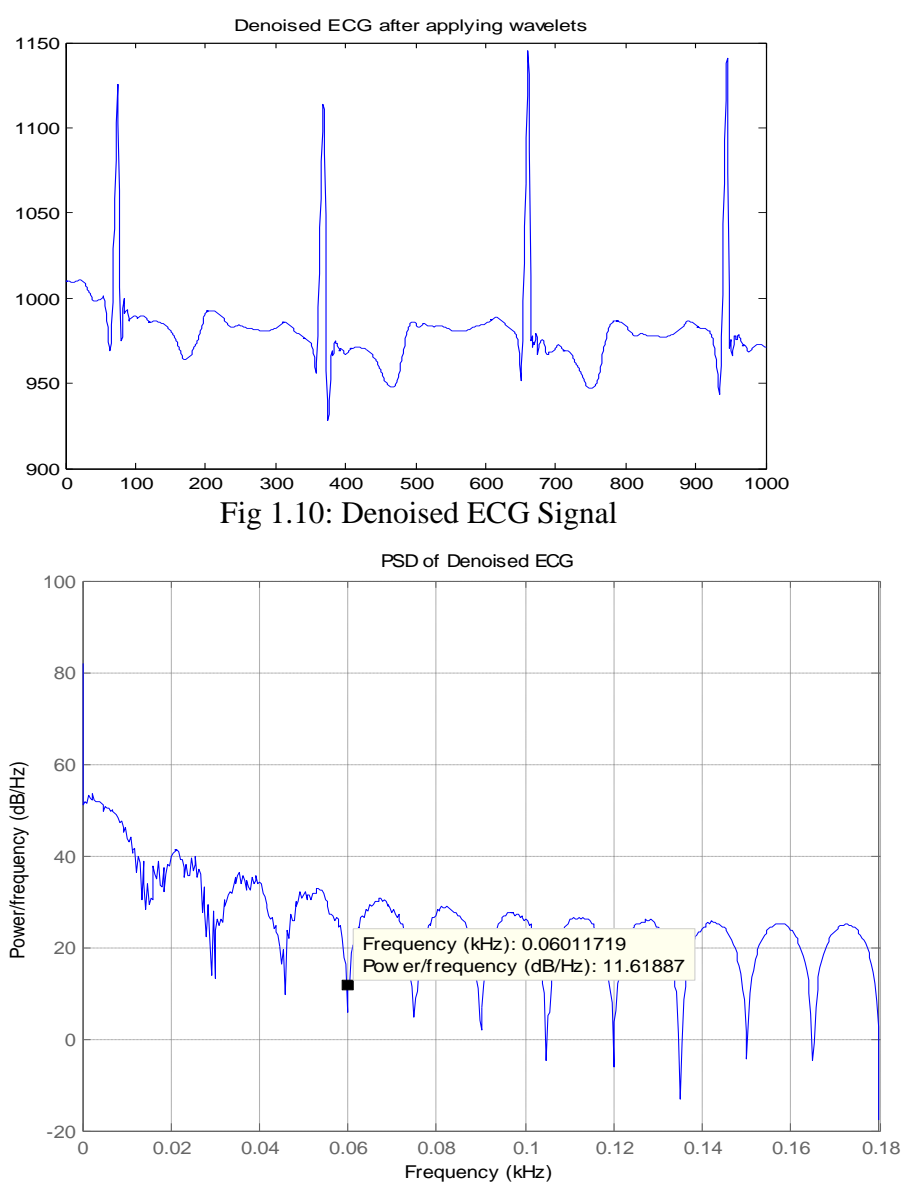

Fig 1.11: PSD of Denoised ECG Signal

A sample signal is taken and its PSD have been obtained (Fig.1.8, 1.9). After that Wavelet has been applied on the signal and PSD has been obtained again (Fig. 1.10, 1.11). 


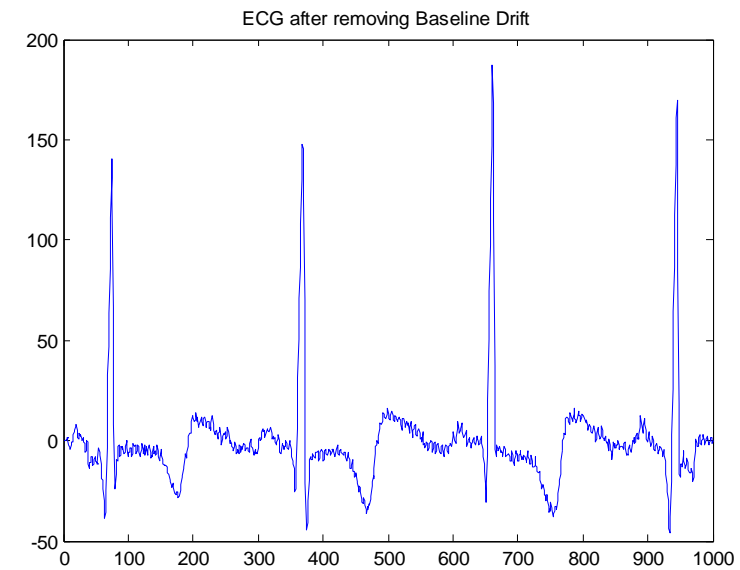

Fig 1.12: ECG after removing Baseline drift

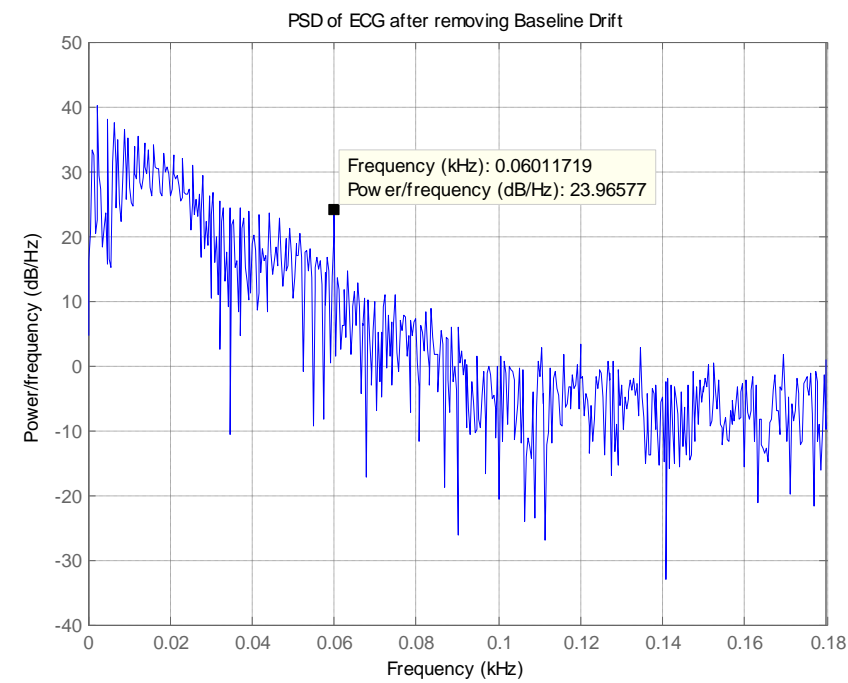

Fig 1.13: PSD of ECG after removing Baseline drift The power spectral density has been reduced at $60 \mathrm{~Hz}$. Hence overall denoising of the ECG signal has reduced PSD at $60 \mathrm{~Hz}$.

In the second stage, Wavelets are applied on the ECG signals after removing Baseline drift. With the help of Moving Average method, and PSD of the signal thus obtained has been noted (Fig.1.12,1.13).

Then Wavelets are applied and again PSD has been obtained (Fig. $1.14,1.15)$. The results of sample data are as shown in Table 2.

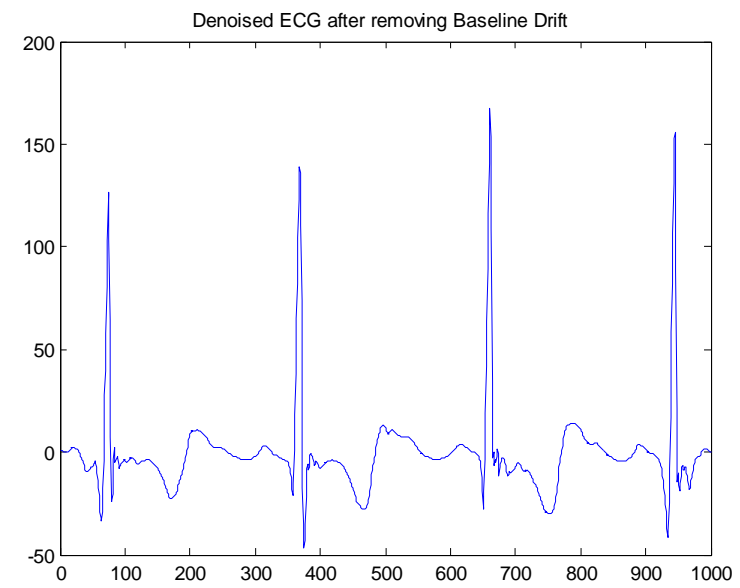

Fig 1.14: Denoised ECG Signal after removing Baseline drift

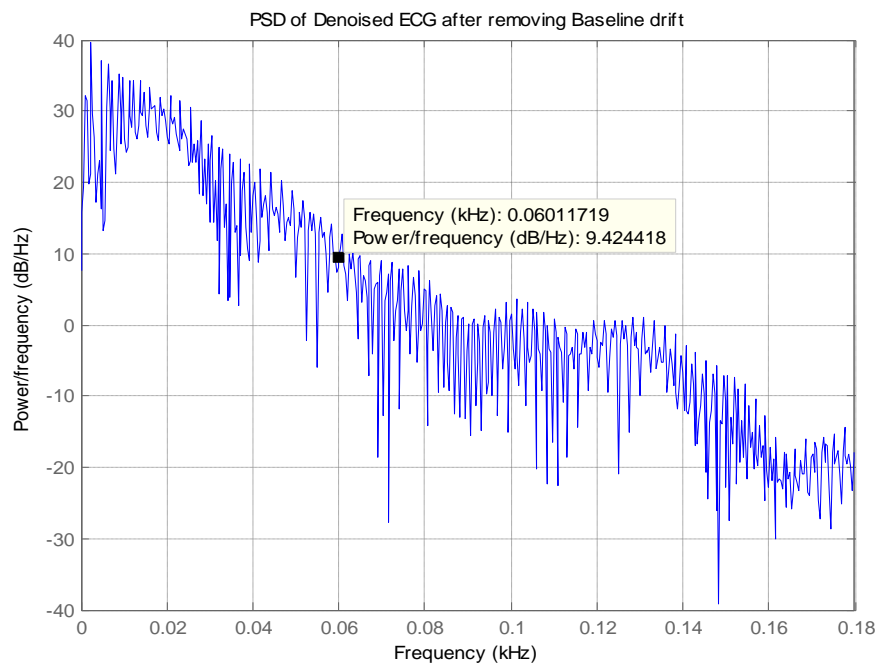

Fig 1.15: PSD of Denoised ECG Signal after removing Baseline drift

A little change has been observed in the PSD of the resultant signal from which baseline has been removed but considerable change is there in PSD after applying Wavelets.

In the third step, Wavelets are applied on ECG signals after removing the baseline drift and application of IIR Notch Filter. The PSDs obtained are compared as shown in Table 3.

Table 3 :

\begin{tabular}{|l|l|l|l|}
\hline $\begin{array}{l}\text { S } \\
\text { No }\end{array}$ & $\begin{array}{l}\text { Sample } \\
\text { Data }\end{array}$ & $\begin{array}{l}\text { PSD after } \\
\text { Baseline Drift } \\
\text { and applying } \\
\text { Notch }\end{array}$ & $\begin{array}{l}\text { PSD of Denoised } \\
\text { ECG(After removing } \\
\text { Baseline Drift and } \\
\text { applying Notch) }\end{array}$ \\
\hline 1 & Sample 1 & -8.148651 & 2.157648 \\
\hline 2 & Sample 2 & -15.333 & 4.783006 \\
\hline 3 & Sample 3 & -15.5721 & -20.78554 \\
\hline
\end{tabular}




\begin{tabular}{|l|l|l|l|}
\hline 4 & Sample 4 & -7.969224 & 8.180872 \\
\hline 5 & Sample 5 & 6.486545 & 7.212292 \\
\hline 6 & Sample 6 & 5.85502 & 10.60792 \\
\hline
\end{tabular}

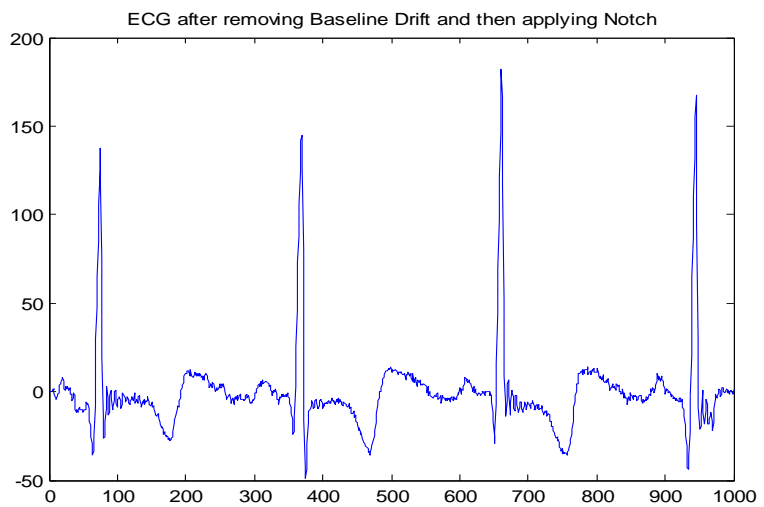

Fig 1.16: ECG after removing Baseline drift and then applying Notch

From Fig. 1.16, Fig. 1.17, the $60 \mathrm{~Hz}$ noise reduces to greater extent but there are some ripples introduced in the ECG Signal after applying Notch filter. The above ECG signals have shown a ripple free ECG (Fig. 1.18)

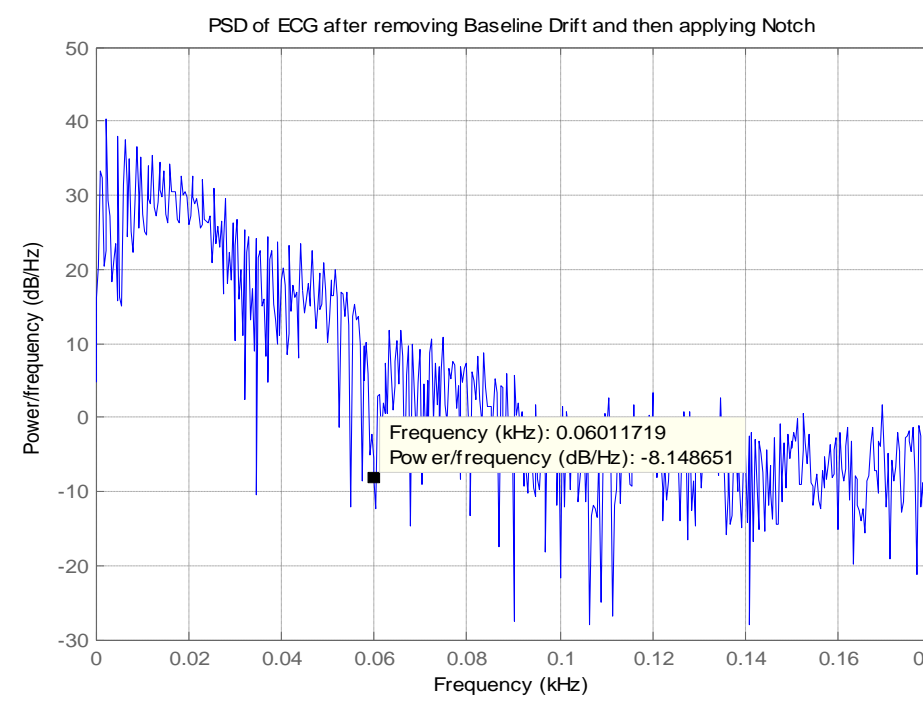

Fig 1.17 : PSD of ECG after removing Baseline drift and then applying Notch

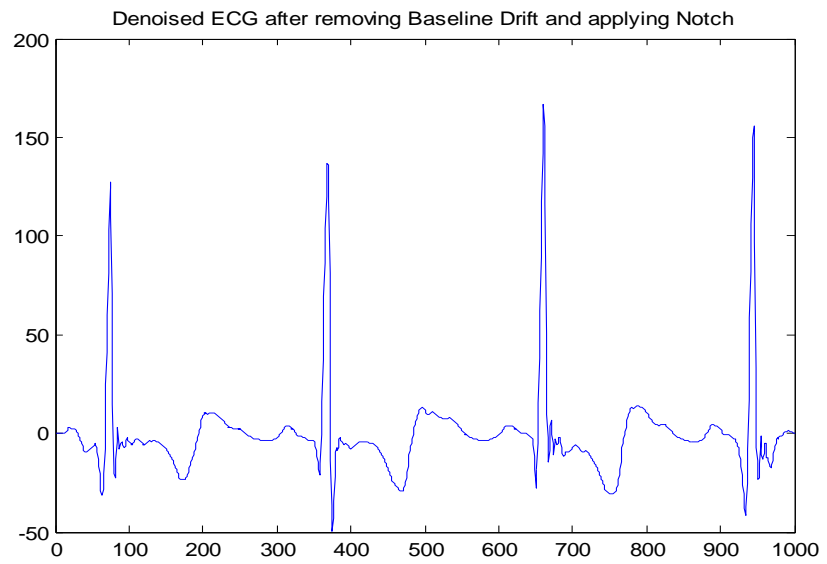

Fig 1.18: Denoised ECG Signal after removing Baseline drift and applying Notch

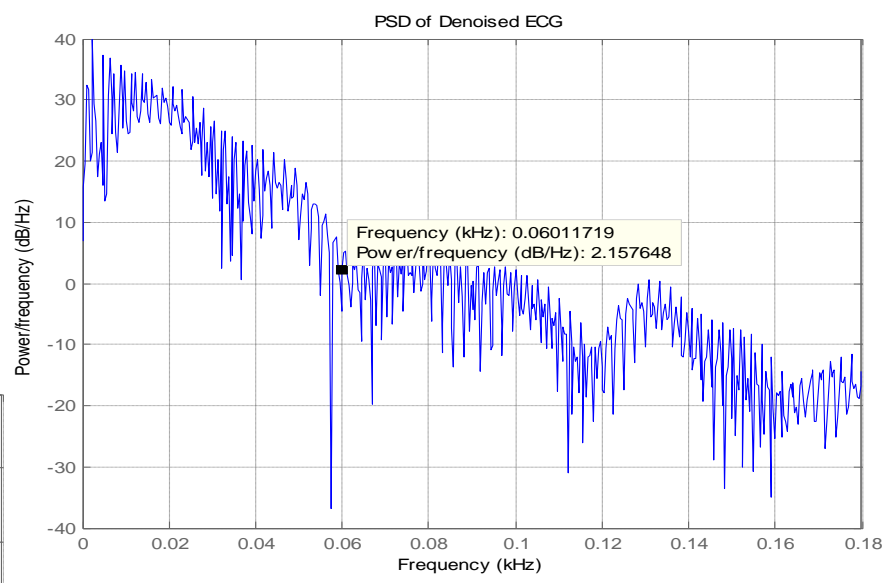

Fig 1.19: PSD of denoised ECG after removing Baseline drift and applying Notch

\section{CONCLUSIONS}

The IIR notch applied directly on the ECG sample have more ringing effect as QRS complex often poses a large unwanted impulse to the filter. When the same filter is applied to the ECG signal which has been smoothed first i.e. having the baseline wander removed, lesser ringing effect has been observed. The wavelets applied in the final stage have removed that ringing effect also, it is clear that the noise has been reduced at $60 \mathrm{~Hz}$. 0 . The filter proposed in this paper has fewer coefficients and hence lesser computation time. So the above combination of three methods, Moving Average, Notch filtering and Wavelets, is more suitable and efficient for real time processing of ECG signals.

\section{REFERENCES}

[1] E.Cromwell, F.J.Weibell, E.A.Pfeiffer, 'Biomedical Instrumentation and Measurements', $2^{\text {nd }}$ Ed., Pearson Education.

[2] http://en.wikipedia.org/wiki/File:SinusRhythmLabels.svg

[3] Alireza K Ziarani, Adaibert Konrad, 'Non linear Adaptive method of elimination of power line interference in ECG signals', IEEE Transactions on Biomedical Engg, Vol.49, No.6, June 2002, pp. 540-544. 
[4] S.Pooranchandra, N.Kumaravel, 'A novel method for elimination of power line frequency in ECG signal using hyper shrinkage function', Digital Signal Processing, Volume18, Issue 2, March 2008, pp. 116-126.

[5] Santpal Singh Dhillon, Saswat Chakrabarti, 'Power Line Interference removal From Electrocardiogram Using A Simplified Lattice Based Adaptive IIR Notch Filter', Proceedings of the $23^{\text {rd }}$ Annual EMBS International conference, October 25-28, Istanbul, Turkey, 2001, pp.340712.

[6] Mahesh S.Chavan, R.A.Aggarwala, M.D.Uplane, 'Interference reduction in ECG using digital FIR filters based on Rectangular window', WSEAS Transactions on Signal Processing, Issue 5, Volume 4, May 2008, pp.340-49.

[7] Chavdar Levkov, Georgy Mihov, Ratcho Ivanov, Ivan Daskalov, Ivaylo Christov, and Ivan Dotsinsky, 'Removal of power line interference from the ECG : a subtraction Procedure', Biomed Eng Online, 2005;4;50, published online 2005 August 23 doi: 10.1186/1475-925X-4-50.

[8] Ziarani AK, Konard A, 'A nonlinear adaptive method of elimination of power line interference in ECG signals', IEEE Trans BiomedEng; 49(6), Jun 2002, pp 540-47.

[9] LIN Yue-Der, YU HEN HU, 'Power-Line Interference Detection and Suppression in ECG Signal Processing', IEEE Transactions on Biomedical Engineering ISSN 0018-9294, 2008, vol. 55, pp. 354-357.
[10] G. Mihov, Iv Dotsinsky, Ts Georgieva, 'Subtraction procedure for powerline interference removing from ECG: improvement for non-multiple sampling', Journal of Medical Engineering \& Technology, Volume 29, Issue 5, September 2005, pp $238-243$.

[11] K.Daqrouq, 'ECG baseline Wandering Reduction Using Discrete Wavelet Transform', Asian Journal of Information Technology 4(11), 2005, pp. 989-995.

[12] P.E.Tikkane, 'Non linear wavelet and wavelet packet denoising of electrocardiogram signal', Biological Cybernetics, Vol 80, No 4, April 1999, pp. 259-267.

[13] Rangarej M Rangayyan, Biomedical Signal Analysis - A case study approach, Wiley Interscience.

[14] DATAQ Instruments.

[15] S.Z.Mahmoodabadi, A.Ahmadian, M.D.Abolhasani, 'ECG Feature Extraction UsingDaubechies Wavelets', Proceedings of Fifth IASTED Interntional Conference Visualization, Imaging and Image Processing, September 7-9, 2005, Benidorm, Spain, pp. 343-348.

[16] Ju-Won-Lee, Gun-Ki Lee, 'Design of an Adaptive Filter with a Dynamic Structure for ECG signal Processing', International Journal of Control, Automation and Systems, vol.3, no.1, March 2005, pp. 137-42. 